Dieses Dokument ist eine Zweitveröffentlichung (Postprint) /

This is a self-archiving document (accepted version):

Milan Pešić, Uwe Schroeder, Stefan Slesazeck, Thomas Mikolajic

Comparative Study of Reliability of Ferroelectric and AntiFerroelectric Memories

Erstveröffentlichung in / First published in:

IEEE transactions on device and materials reliability. 2018, 18(2), S. 154-162 [Zugriff am: 18.08.2021]. IEEE. ISSN 1558-2574.

DOI: https://doi.org/10.1109/TDMR.2018.2829112

Diese Version ist verfügbar / This version is available on:

https://nbn-resolving.org/urn:nbn:de:bsz:14-qucosa2-767094 


\title{
Comparative Study of Reliability of Ferroelectric and Anti-Ferroelectric Memories
}

\author{
Milan Pešić ${ }^{(}$, Uwe Schroeder $\left.{ }^{(}\right)$, Stefan Slesazeck, and Thomas Mikolajick
}

\begin{abstract}
With the discovery of the ferroelectric (FE) properties within $\mathrm{HfO}_{2}$, the scaling gap between state-of-the-art technology nodes and non-volatile memories based on FE materials can be bridged. In addition to non-volatility, new memory concepts should guarantee sufficient endurance and operation stability. However, in contrast to optimized perovskite based FEs, binary oxide based FE memories still show changes in the memory window (MW) followed by either hard breakdown or closure of the MW. Recently, we have shown that anti-FE (AFE) materials exhibit very stable and significantly higher endurance with respect to the $\mathrm{FE}$ counterparts. Inspired by the robustness and remarkable cycling performance of the AFE materials, we analyze the remaining reliability aspects of these devices. By characterizing the pure film properties of capacitor stacks and switching performance when integrated into devices, we compare and investigate temperature stability, imprint, retention, and variability of both FE and AFE memories. We investigate if the lower energetic barrier to be overcome together with partial switching and lower switching induced stress are responsible for the higher endurance of the AFE with respect to the FE based memories. By utilizing charge trapping and charge pumping tests together with leakage current spectroscopy in combination with comprehensive modeling we check that assumption. Moreover, we identify the interfacial buffer layer as the weakest link of these devices.
\end{abstract}

Index Terms- $\mathrm{HfO}_{2}$, FeRAM, AFE-RAM, FeFET, ferroelectric-memory, reliability.

\section{INTRODUCTION}

D UE TO their limited scalability perovskite based ferroelectric memories are limited to niche markets [1], [2]. Ferroelectric (FE) binary oxides, based on $\mathrm{HfO}_{2}$ and $\mathrm{ZrO}_{2}$ [3], revived the interest in FE memory concepts. It also triggered the research in different application fields like steep-slope devices [4]-[6] and neuromorphic [7] applications. The presence of high-k solutions in semiconductor industry and especially $\mathrm{HfO}_{2}$ as the gate dielectric in state-of-theart logic nodes [8], made the integration of FE hafnia into the gate stack of the transistor a straightforward approach.

Manuscript received December 4, 2017; revised February 22, 2018; accepted April 18, 2018. Date of publication April 20, 2018; date of current version June 5, 2018. This work was supported in part by the European Fund for regional Development EFRD and in part by the Free State of Saxony, Europe supports Saxony. (Corresponding author: Milan Pešić.)

M. Pešić, U. Schroeder, and S. Slesazeck are with Namlab gGmbH, 01187 Dresden, Germany (e-mail: milan.pesic@namlab.com).

T. Mikolajick is with Namlab gGmbH, 01187 Dresden, Germany, and also with the Chair of Nanoelectronic Materials, Technical University of Dresden, 01187 Dresden, Germany.

Color versions of one or more of the figures in this paper are available online at http://ieeexplore.ieee.org.
This allowed continuation of the scaling of CMOS compatible FE memories [9], [10]. Furthermore, this discovery bridged the scaling gap between state-of-the-art technology nodes and FE memories. However, the reliability issues in binary oxide based FE memories still need to be fully solved. Hafnia based ferroelectrics show an endurance [11]-[14] that is significantly lower than state-of-the-art optimized perovskite based equivalents. Due to the high coercive field and the necessary operation at high electric fields, FE hafnia suffers from breakdown and closure of the memory window (MW). Moreover, when integrated into a ferroelectric field effect transistor (FeFET) device, charge trapping may lead to an even faster closure of the memory window compared to a metal / ferroelectric / metal capacitor [14].

We previously showed [15]-[17] that non-volatility can be achieved in anti-ferroelectric (AFE) materials if asymmetric electrodes are used. This enables devices that show higher endurance strength compared to their FE counterparts. The obtained gain can even fulfill the endurance requirements of a non-volatile random access memory. To analyze the advantages and disadvantages of AFE and FE memory solutions and benchmark their performance against the reliability targets of 10-year retention, $10^{12}$ program/erase (PRG/ERS) cycles, etc., an in-depth comparative/reliability assessment is mandatory. The correlation of the physical mechanisms with the device behavior is essential to understand the device and enable the engineering of superior devices. The paper is organized as follows: In the first part device fabrication and physics of the memory effect in both AFE and FE materials are revisited. In the next step, material properties such as domain and switching distribution, coercive field and local bias distribution within the AFE- and FE capacitor and their impact on the variability will be analyzed. Finally, properties and reliability aspects of integrated $28 \mathrm{~nm}$ FE- and AFE- $\mathrm{HfO}_{2}$ based field effect transistors (FETs) are compared.

\section{FABRICATION Flow AND DEVICE StaCK}

\section{A. Ferroelectric and Anti-Ferroelectric Capacitors}

$10 \mathrm{~nm}$ thick $\mathrm{ZrO}_{2}$ (AFE) or $\mathrm{Si}$ doped $\mathrm{HfO}_{2}$ (FE) films are deposited on a TiN bottom electrode. The AFE films are covered by a $\mathrm{RuO}_{\mathrm{x}}$, while $\mathrm{FE}$ layers by a TiN top electrode to form metal-insulator-metal (MIM) stacks. In the next step, capacitors are annealed in nitrogen atmosphere at $800{ }^{\circ} \mathrm{C}$. This crystallization step is required for stabilization of the tetragonal (in $\mathrm{ZrO}_{2}$ ) and the orthorhombic (in $\mathrm{Si}: \mathrm{HfO}_{2}$ ) phase responsible for the AFE and FE properties, respectively. More 
details about the processing can be found in [13] and [16]. These capacitor structures will be used as test vehicles in Section IV when investigating and comparing material properties of (A)FE films. In addition to planar capacitor, fully integrated $6 \mathrm{~F}^{2}$ DRAM cells realized in buried wordline technology with trench $\mathrm{ZrO}_{2} / \mathrm{Al}_{2} \mathrm{O}_{3} / \mathrm{ZrO}_{2}(\mathrm{ZAZ})$ capacitors with high aspect ratios (32:1) are characterized. Details about the used dynamic random access memory (DRAM) technology can be found in [18].

\section{B. Ferroelectric and Anti-Ferroelectric Field Effect Transistors}

In the second part of the study fully integrated FeFET multi-structure devices that share the gate and bulk terminal are compared. Previously, it has been shown that the FE and AFE properties of the material can be tailored by tuning the $\mathrm{Si}$ doping concentration within the $\mathrm{HfO}_{2}$ film [19]. Devices investigated in this study are fabricated in $28 \mathrm{~nm}$ high-k metal gate (HKMG) technology with a gate stack comprising an $0.8 \mathrm{~nm}$ interfacial SiON layer, $9 \mathrm{~nm}(\mathrm{~A}) \mathrm{FE} \mathrm{HfO}_{2}$ layer doped with (5.7) $4.4 \mathrm{~mol} \% \mathrm{SiON}$ and an $8 \mathrm{~nm}$ TiN metal layer. More details about these devices are reported in [20].

\section{FundAMENTALS OF FERROELECTRIC AND ANTI-FERROELECTRIC MEMORIES}

Based on the Landau theory the energy potential of a FE can be represented as a system with two stable energy minima (see Fig. 1a) separated by a potential barrier. This barrier between the two stable minima guarantees non-volatility of the polarization. In contrast to FEs, AFEs are characterized by a single minimum and two symmetric kinks. Application of an external field transforms one of the energy potential kinks into a 'FE like' two well system with a lower barrier compared to the FE case. In contrast to the potential minima in a FE, these minima of the AFE are volatile (see Fig. 1b) and will be lost when the external electric field is removed. Previously Pešić et al. [15], [16] suggested, that utilization of electrodes with asymmetric workfunctions would generate a built-in bias which centers one of the branches of the AFE and hence enables non-volatility (see Fig. 1c). The gain of this approach is reflected through the fact that the effective field required for switching i.e., coercive field $\left(E_{c}\right)$ is halved, hence enabling lower operating voltages and lower fields during cycling, resulting in higher endurance.

Both FE and AFE materials can be implemented in 1T (1 transistor) and 1T-1C (1 transistor- 1 capacitor) memory cells. In the $1 \mathrm{~T}-1 \mathrm{C}$ configuration, during reading the capacitor needs to be biased and the bitline is charged either by the available switchable polarization or only by the displacement current of the capacitor depending on the fact if the capacitor is switched or not. In the 1T configuration the channel conductivity is altered by the polarization state. Hence the threshold voltage of the transistor is altered. Details can be found in [20] and [21].

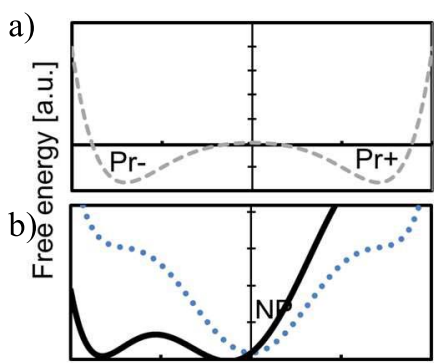

Polarization [a.u.]

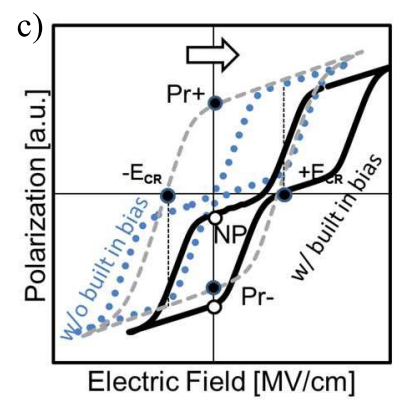

Fig. 1. Energy landscape and corresponding polarization hystereses of a FE (dashed gray) and an AFE(blue dotted) as well as the biased AFE (solid black). a) Free energy as a function of the polarization for a FE material. b) Free energy as a function of the polarization for a AFE (blue dotted) material and a biased AFE material (solid black). c) Polarization hystereses of a capacitor using a FE (dashed grey line), an AFE (dotted blue line) and a biased AFE (solid black line) material.

\section{Material and Reliability Aspects of FERROELECTRICS AND ANTI-FERROELECTRICS}

Before discussing fully integrated devices and reliability aspects originating from the integration and operation scheme, pure material properties of AFE and FE films, as main functional blocks, should be investigated. Hence, in this section endurance and retention of the FE and AFE capacitors will be compared and analyzed. Discussion of these two classic reliability features, will be accompanied with discussion of imprint as an additional aspect of FE and AFE based memories.

\section{A. Endurance}

Compared to perovskite based FE memories [12]-[14], binary oxide based equivalents are still inferior in terms of cycling stability. The classical fatigue phenomenon which is reflected in the continuous reduction of the memory window for high cycle counts, is not controlled in a similar way. Additionally, because of the very high coercive field of ferroelectric hafnia, endurance is often limited by breakdown of the ferroelectric layer. The memory window closure by fatigue can directly be observed in ferroelectric capacitors [12]-[13]. In ferroelectric field effect transistors (FeFET) in most cases charge trapping is dominating the window closure [11]. Within the endurance characteristics of the typical FE capacitor (FeCap), before breakdown two stages can be identified: a) wake-up i.e., opening of the MW by depinching of the hysteresis loop and b) polarization fatigue i.e., reduction of the available switchable polarization (collapse of the hysteresis loop) [13], [22]. Details about possible mechanisms responsible for this MW changes are reported in [13] and will be just briefly reviewed. The reduced coercive field of the AFE with respect to the FE equivalents enables significant reduction of the operation voltage and hence impedes stress induced defect generation and drastically increases the stress time before breakdown. Nonetheless, to generate equal stress conditions as well as to induce equal dielectric degradation, both AFE and FE capacitors (of the same thickness) are stressed with the same voltages. As it can be seen from Fig. 2a (triangles), AFE capacitors are characterized by nearly 

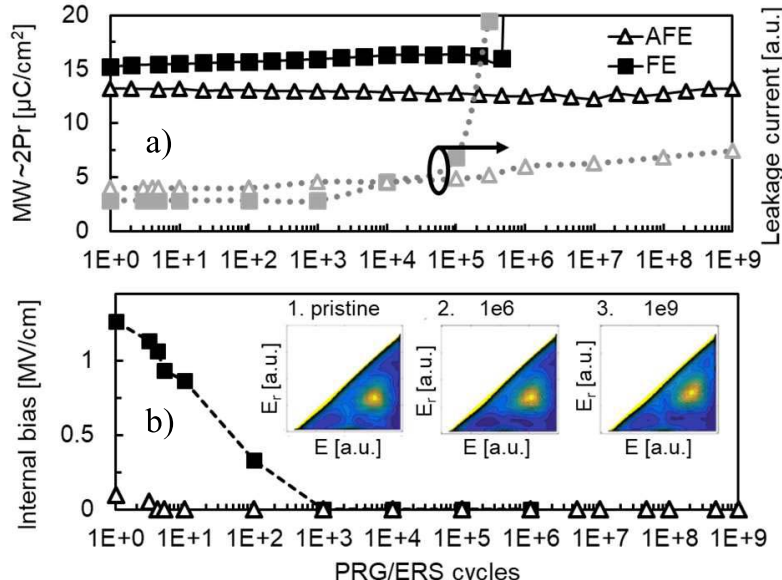

Fig. 2. Comparison of the field cycling behavior of a FE (squares) and AFE (triangles) capactior. a) MW and $2 \mathrm{P}_{r}$ evolution (black simbols and solid lines, left axis) together with the evolution of the leakage current (grey simbols and dashed lines, right axis) extracted at $1 \mathrm{~V}$ of the AFE (triangles) and FE (squares) capacitor. b) Local internal bias field as a function of stress cycles. The insets show the Preisach densty distribution plots in function of applied and reversal field $\mathrm{E}, \mathrm{E}_{\mathrm{r}}$ respectively for 1. a pristine sample; and after 2. $10^{6}$ and $3.10^{9}$ PRG/ERS cycles. The PRG/ERS operation conditions used are $\pm 3 \mathrm{~V}$ at $100 \mathrm{kHz}$

wake-up and fatigue free endurance behavior compared to the FE equivalents.

One disadvantage of the $\mathrm{HfO}_{2}$ based $\mathrm{FE}$ materials is the high coercive field of $1-\mathrm{MV} / \mathrm{cm}$ (and higher) which is much closer to the breakdown field compared to perovskite materials. Accordingly, continuous switching is generating defects which can act as trap centers that slow-down the switching dynamics and cause domain-pinning, yielding a closure of the MW [12], [13]. Further, FE-switching induced defect generation is responsible for increase of the leakage current as seen in Fig. 2a which, in FE case, finally results in hard breakdown of the device. The wake-up behavior is caused by non-uniform properties of the films i.e., non-uniform vacancy and charge distribution as well as a polyphase presence in the film which gets more uniform with field cycling [13], [22]. Further, these non-uniformities act as dead (non-switching) layers that generate a depolarization field. All these nonuniformities together are responsible for the formation of local internal bias fields that can be studied by means of first order reversal curves (FORC) [23]. The extracted local internal bias is shown in Fig. 2b. As in previous studies, we show that during the wake-up stage the bias steadily diminishes together with a decreasing depolarization field [24]. Moreover, FORC can be used to depict the domain switching density which, in case of pristine $\mathrm{FE}$, is characterized by two distributions separated by an internal bias. Here, we plot an AFE domain distributions in three different stages: 1) pristine 2) after $10^{6}$ cycles and after $10^{9}$ cycles (see inset of Fig. 2). In contrast to FEs, which are typically characterized by a phase mixture which contributes to film non-uniformity and generates local internal bias fields, no local internal bias field (see Fig. $2 \mathrm{~b}$ triangles) is observed for the AFE case. This is reflected by a single distribution observable in the Preisach switching plain (see inset of Fig. 2b). Compared to the pristine stage of FE

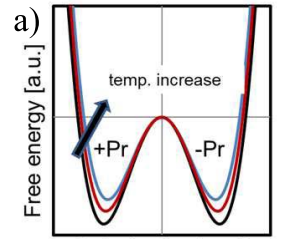

b) Polarization [a.u.]

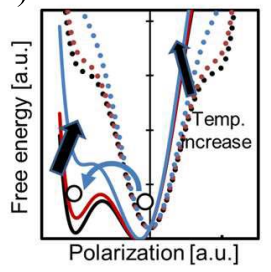

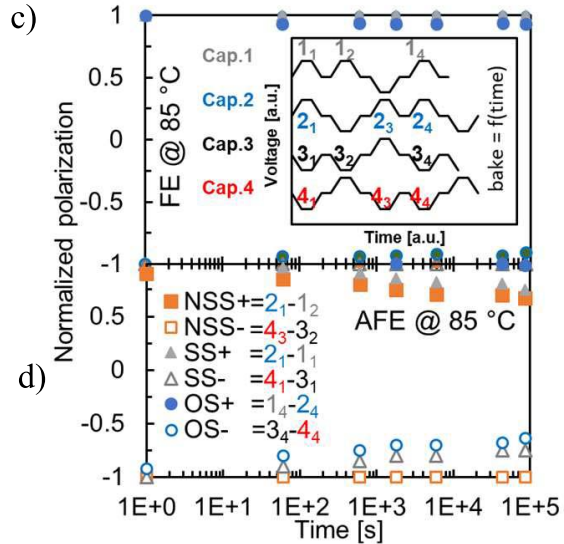

Fig. 3. Illustration of free energy evolution with increasing temperature of a) FE and b) AFE based devices. Retention data at $85^{\circ} \mathrm{C}$ for the dfferent states descriped in the text measured on a c) FE and d) AFE capacitor. Four capacitors are used for each test according to [26]. The inset in figure c) shows the pulse train used for the retention assessment of the four ferroelectric capacitors. Within the pulse sequence pulses were delayed by $1 \mathrm{~ms}$. After the application of the pulse sequence test structures were baked with increasing time steps. The calculation of retention values (NSS, SS and OS) is given in the legend of the figure d) and its values are obtained from the respective integrated current response.

films where typically a monoclinic/tetragonal/orthorhombic phase mixture is present, the whole film is tetragonal and hence more uniform in the AFE case. Moreover, simulation results [25] predict a favorable stabilization of the AFE tetragonal phase for a film thickness below $5 \mathrm{~nm}$ which would be another advantage of AFE with respect to FE devices for sub-20nm nodes. Finally, a reduced degradation of dielectric caused by AFE-switching is reflected through the insignificant increase of the leakage current (compared to $\mathrm{FE}$ equivalent) with cycling (see Fig. 2a).

\section{B. Retention}

Beside the high endurance strength NVM should provide long enough retention. Retention of the FE and AFE materials is evaluated by using four discrete capacitors [26] and is based on a modified positive-up negative-down (PUND) sequence, with pulses having a $1 \mathrm{~ms}$ spacing within the sequence. Analogous to the PUND tests, the resulting current is dependent on the voltage history and the previous state in which the capacitor resided before the measurement. In that manner the ratio between the ferroelectric and dielectric components is determined and analyzed. Before a bake step, on each FE and AFE capacitor a designated pulse waveform is applied (see inset of Fig. 3c). Details about the waveform (see inset of Fig. 3c), method in general can be found in [26] whereas the memory state calculation is given as part of the legend of the Fig. 3d. The last pulse of each sequence (see inset of Fig. 3c) was used to set a defined polarization state, after which the sample was stored at different temperatures for different time intervals. After each bake (carried in increasing time steps), the same sequence was applied to the designated capacitors to evaluate the amount of the residing state. Calculated states are opposing-state (OS), same-state (SS) and new samestate (NSS). In the study performed by Mueller et al. [27] 
a)

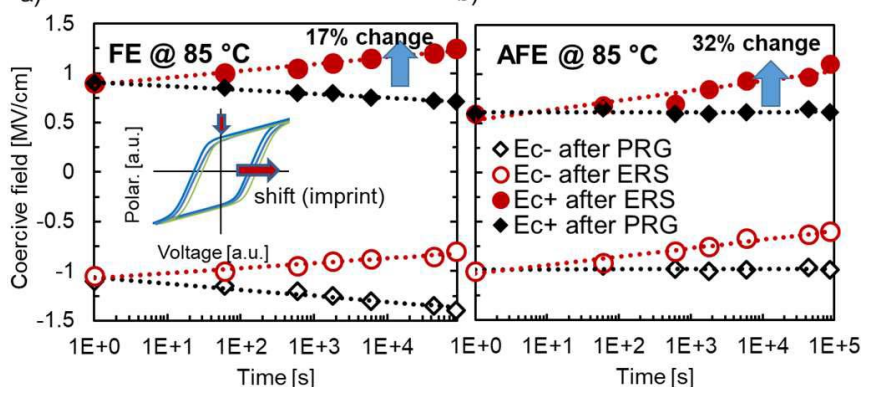

Fig. 4. Shift of positive and negative coercive field of the a) $\mathrm{Si}: \mathrm{HfO}_{2} \mathrm{FE}$ and b) $\mathrm{ZrO}_{2}$ based AFE capacitor structure tested at $85^{\circ} \mathrm{C}$. The inset in a) schematically shows a storage related biasing of the P-V hysteresis due to the imprint. The value [\%] denotes ratio of imprinted state (coercive field shift) and $2 \mathrm{E}_{\mathrm{c}}$ of the device.

negligible retention loss is observed for saturated polarization states up to cumulative testing times of $1000 \mathrm{~h}$ at elevated temperatures of $125^{\circ} \mathrm{C}$.

We repeated the experiments for the for the $\mathrm{FE} \mathrm{Si:HfO}$ based and AFE $\mathrm{ZrO}_{2}$-based capacitors. From the energetic perspective, artificially generated double well potential (due to the built-in bias) of AFE is not centered around the origin (zero polarization) (Fig. 3b) and hence compared to FE is more vulnerable to high temperatures as shown by simulation and experiments [20], whereas a double well of FE is centered around the origin (Fig. 3a). As anticipated, the reduction of the switching barrier of $\mathrm{AFE}\left(\mathrm{E}_{\mathrm{c}}\right.$ of $\left.0.75 \mathrm{MV} / \mathrm{cm}\right)$ results in retention only up to $85{ }^{\circ} \mathrm{C}$ compared to Muellers experiments performed on $125^{\circ} \mathrm{C}$ and higher (see Fig. $3 \mathrm{c}-\mathrm{d}$ ).

\section{Imprint}

In addition to previously discussed retention caused by depolarization, the shift of the hysteresis is a specific reliability issue that needs to be evaluated in detail in FE and AFE materials. After setting the devices in a defined (negative or positive) polarization state, a bake step at $85^{\circ} \mathrm{C}$ is performed. Subsequently the bake step is followed by a readout of the polarization-voltage curve and the coercive field shift evolution (compared to initial reference position) is monitored (see Fig. 4). The mechanisms behind the imprint can be understood as follows: When a ferroelectric material resides in a positive or a negative polarization an internally generated bias of the residing state tends to shift the polarization curve along the field axis (see inset Fig. 4a). Charges trapped at one or the other interface to the electrode are assumed to be the origin of the bias creating the voltage shift of the hysteresis. Consequently, the biasing of the P-V can reduce the remanent polarization of one state and hence result in a retention loss or a loss of signal when the device is reprogrammed into the opposite polarization state.

Due to its nature in the AFE only one state is affected since the material has one state with and one state without internal polarization. This is confirmed in our experiments (see Fig. 4b). In contrast, the FE film has a positive and a negative polar state in which the system can reside. Both states could induce biasing of the P-V curve. Still, like in retention
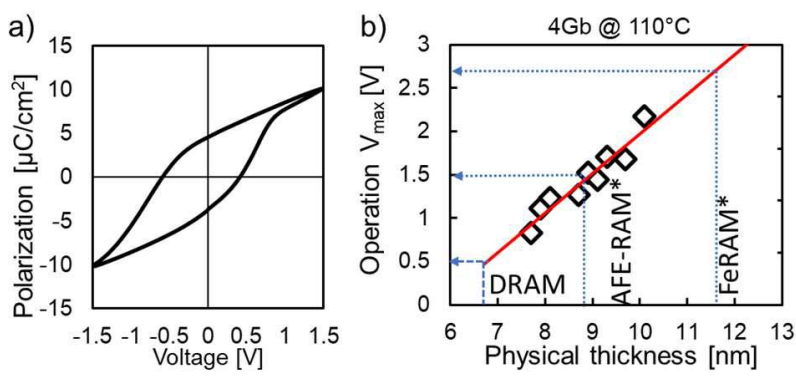

Fig. 5. (a) Polarization voltage characteristics of an AFE-RAM with different workfunction values for the electrodes resulting in a shift of the hysteresis as explained by figure 1c). b) Maximum operating voltage vs. phasical-thickness for a $\mathrm{ZrO}_{2}$ based dielectric with TiN electrodes in a 3D DRAM configuration. The 10 year lifetime target is indicated for different memory concepts realized using the same material system. The values for AFE and FE RAM are estimates calculated based on the typical coercive fields and operation voltages of the capacitors used in this sudy.

test, the AFE devices show a stronger shift of the affected, negative state compared to the FE equivalent. Moreover, an additional effect originating from the workfunction difference might appear during the operation of the device [28].

\section{Leakage Decrease and 3D Integration}

Similar to $\mathrm{ZrO}_{2}$ based DRAM dielectrics [29], [30] an amorphous interlayer could be used in both a FE and AFE capacitor stack to decrease the leakage current and increase the reliability of the device. A sub-nanometer thick interlayer blocks propagation of the grain boundaries from the bottom to the top electrode within this film and does not disturb the polarization-switching properties of the film in both AFE and FE capacitors [20], [31]. To alter a state of the FE memory, a coercive field that is below but close to the breakdown field of the dielectric must be overcome. Therefore, the application of a very high field that is required for consecutive switching of the devices cumulatively deteriorates the dielectric and increases the power consumption of the chip. Usage of an AFE films and asymmetric electrodes results in centering of one of the AFE loops and yields a reduction of the field required for switching by about 30 to $50 \%$ (see Fig. 5a). To realize a scaled $1 \mathrm{~T}-1 \mathrm{C}$ memory cell and at the same time provide sufficient polarization charge, independent on the technology (FE or AFE), integration into 3D capacitors with high aspect ratio is required. In the AFE solution only half of the switchable polarization is used and therefore an even higher aspect ratio is required. As a test vehicle fully integrated $6 \mathrm{~F}^{2}$ DRAM cells realized in buried wordline technology with high aspect ratios (32:1) are used. To extrapolate the lifetime of the device to use conditions different positive voltages are applied at elevated temperatures [30] in a positive constant voltage stress (CVS) experiment. The lifetime for different ZAZ stack thicknesses with two TiN electrodes was determined. Based on this experiments, operating conditions and lifetime specification for a $4 \mathrm{~Gb}$ memory cell are calculated (See Fig. 5b). It can be seen that for extrapolated operating conditions of a $2 \mathrm{~V}$ stack thickness of $10 \mathrm{~nm}$ would be sufficient and a further reduction of the switching condition to $1.5 \mathrm{~V}$ would allow the usage of $9 \mathrm{~nm}$ thick film stacks. 
TABLE I

PARAMETERS USED FOR Simulations

\begin{tabular}{|c|c|c|c|c|}
\hline$d_{I F}[n m]$ & $d_{\text {Hfo2 }[\mathrm{nm}]}$ & $\boldsymbol{k}_{\mathrm{SiON} /(\mathrm{HfO} 2)}$ & $\operatorname{Pr}(\mathrm{Ps})\left[\mu \mathrm{C} / \mathrm{cm}_{2}\right]$ & $E_{c}[M V / c m]$ \\
\hline 0.8 & 10 & $5.3(30)$ & $10(15.1)$ & 1.3 \\
\hline
\end{tabular}
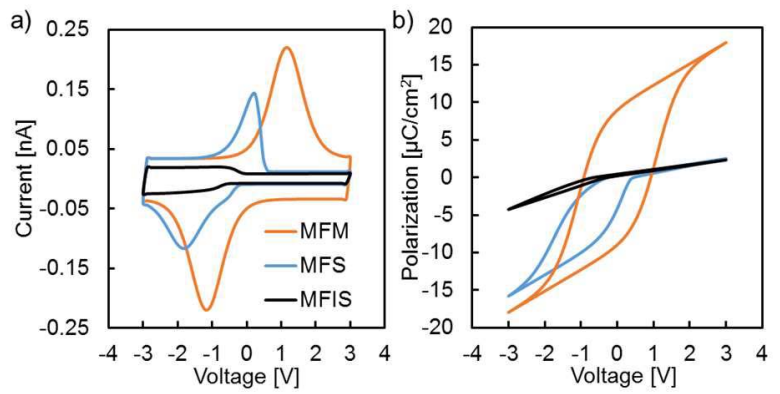

Fig. 6. Simulated a) I-V and b) P-V characteristics of MFM, MFS and MFIS device for a fixed maximum applied voltage of $3 \mathrm{~V}$. The detailed data of the structureare given in table I.

\section{Ferroelectric and Anti-Ferroelectric FIELD EFFECT TRANSISTORS}

The next section of the study is devoted to the reliability aspects of previously discussed materials exhibiting AFE and FE properties when integrated into the gate stack of devices fabricated in $28 \mathrm{~nm}$ HKMG technology. The attractiveness of a FeFET as promising emerging memory solutions lays in its small cell size [11]. The ultra-low power required for writing of a nonvolatile bit is like in the capacitor based concept, with the additional advantage of a nondestructive read [9], [10], [21]. In addition to FeFET devices, metal-ferroelectric-insulator-semiconductor (MFIS) capacitor structures will be simulated.

\section{A. From MFM via MFIS to FeFET: Voltage Divider}

To better understand the gate stack of a FeFET and its reliability aspects, the transition between the MFM capacitor and a FeFET, as well as the emerging depolarization field will be addressed. For this purpose, the model of the MFM capacitor [13], [20], [32] reported earlier is extended to a metal FE semiconductor (MFS) structure. As in the MFM study, the history dependent behavior of the FE switching is realized by utilizing a Preisach hysteresis model. This model is readily available within Sentaurus Device simulator as described in detail in [20] and [32]. Details of the FE implementation and multi-granularity of the model can be found in [13] and [20]. To simulate the transient I-V and corresponding P-V characteristics, a triangular excitation voltage is applied to the MFM, MFS and MFIS devices. Parameters used for the simulation of these different stacks are listed in Table I.

In Fig. 6 it can be seen that in contrast to the symmetric switching peaks in the transient I-V characteristic of a MFM device, the introduction of a silicon semiconductor bottom electrode (MFS structure) results in an asymmetric I-V characteristic as well as in a shift of the hysteresis along the voltage axis.
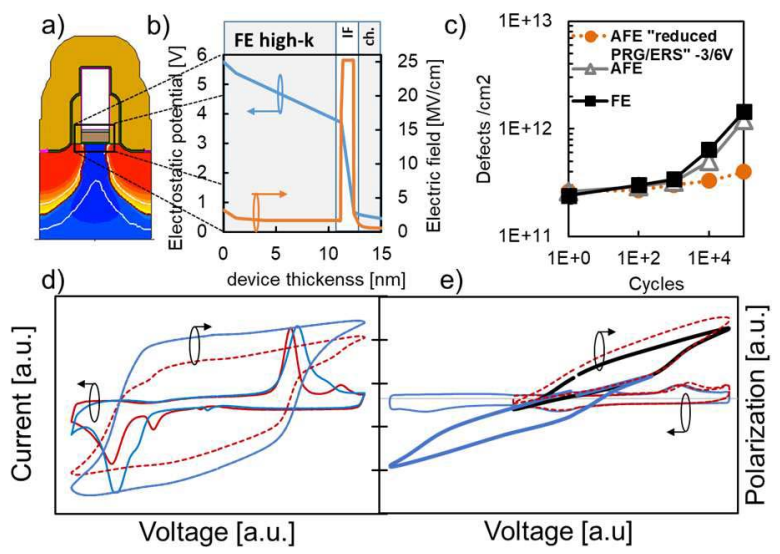

Fig. 7. (a) Simulated device structure and (b) field distribution within the stack for a transistor operting a $+6 \mathrm{~V}$ at the gate. (c) Interface state defect concentration evolution (mean value) as a function of stress condition. I-V and coresponding P-V characteristics of the d) FE and e) AFE based FET.

This built-in bias is generated by the different workfunction values of the TiN top electrode and $\mathrm{Si}$ substrate. Nonetheless, this shift can be reduced by workfunction engineering of the gate metal as well as by doping of the substrate. Additional introduction of an interface buffer layer resulting in a MFIS structure introduces a voltage divider and consequently decreases the total field seen by the switching part of the stack. Reduction of the field over the ferroelectric results in a non-saturating (sub-loop) operation, which can finally lead to a closure of the $\mathrm{P}-\mathrm{V}$ loop $\left(\mathrm{P}_{\mathrm{r}}\right.$ drop) and a reduction of the memory window. Introduction of the interfacial buffer layer generates not only a voltage divider but also increases the depolarization field. The detrimental depolarization field additionally reduces the $\mathrm{P}_{\mathrm{r}}$ and the MW.

A detailed analysis of the reliability aspects of the FeFET stack, such as interface breakdown, depolarization field and charge trapping will be given in Sections V-B to V-D.

\section{B. From MFM via MFIS to FeFET: Interface Breakdown}

As previously discussed, the parasitically generated voltage divider shares a portion of the applied field over the FE and dielectric $\left(\mathrm{SiO}_{2}\right)$ interface capacitances. The ratio between the k-value of the FE high-k and SiON-based interface buffer layer (IBL) determines the portion of the field drop over each of the constituents. As the SiON-based IBL is characterized by a significantly lower k-value compared to the $\mathrm{FE}: \mathrm{HfO}_{2}$, a huge voltage-drop over the IBL is facilitating its degradation. Field and degradation factors are calculated by using a TCAD model of a FeFET that is implemented and simulated in Sentaurus Device. As before, a history dependent FE behavior is emulated using a Preisach model of the hysteresis [13], [32]. A typical PRG operation condition (6V applied on the gate while all remaining terminals are grounded) is simulated and the field distribution over the stack is extracted and shown in Fig. 7b. As anticipated, most of the potential drop occurs over the lower $\mathrm{k}$ IBL, whereas the remaining electric field is seen by the FE high-k. In the following, a cumulative impact of these high fields over the interface will be discussed. 
The influence of the stress induced degradation caused by the PRG and ERS operation is investigated by performing charge pumping $(\mathrm{CP})$ experiments nested between the PRG/ERS stress sequences. The experiment is carried out as follows: the substrate current ( $\mathrm{CP}$ signal) is measured while voltage pulses of fixed amplitude, rise, and fall time are applied to the gate of the transistor. Alongside the pulses applied to the gate electrode, source and drain of the device under test are shorted and connected to ground. Using the same stressing conditions of $\pm 6 \mathrm{~V}$, the evolution of interface states within AFE- and FE- FET is compared. Even though the AFE, as a material, has higher endurance strength compared to a FE, its advantages cannot be materialized when integrated in a FeFET device. When operated at the same PRG/ERS fields the IBL remains the weak link which degrades at a similar pace (see Fig. 7c). The degradation of the IBL fully correlates to the results reported by Yurchuk et al. [11] and originates from continuous back and forth electron injection caused by alternating PRG and ERS pulses. As a consequence, we show that in transient I-V characteristics of the FE and AFE devices (recorded in parallel with stress dependent $\mathrm{CP}$ experiments) a clear increase of dynamic leakage at maximum field and consequent rounding of the hysteresis shape (see Figs. 7d-e) is visible. With degradation of the interface properties, a strong charging of the high-k FE or AFE layer takes place, which accordingly impacts the properties of the device (see Section V-D for details). To estimate the potential endurance gain obtained by integration of the AFE material, the AFE device is cycled in such a way that only the right loop system is used (see Fig. 7e). This unique property of the AFE can be understood as following: AFE is comprised out of two FE subsystems that are biased in opposing directions and independent of each other [15]. Utilization of the "positive" sub-system enables a reduction of the PRG/ERS voltages which reduces the stress over the device and lower degradation of the interface is the result.

Based on this analysis several conclusions can be made. As was demonstrated in [33] the integration of the AFE as the gate-material results in a reduced variability reflected in a narrower switching distribution. The operating voltages can be reduced due to the reduced $E_{c}$, which would yield in devices with higher endurance strength. However, the reduced $\mathrm{E}_{\mathrm{c}}$ would result in a further reduction of the MW that is anyhow rather small in ferroelectric FETs.

\section{From MFM via MFIS to FeFET: Depolarization field}

As pointed out by Ma and Han [34] and discussed in Section V-A, a depolarization field represents one of the biggest challenges for a bilayer structure comprising an active, FE switching and a passive, non-switching layer (e.g., an interfacial layer). The depolarization field represents a field in opposing direction to polarization state that tends to flip the residing state of the ferroelectric (see Fig. 8) and is one of the main causes of the retention loss in these devices [34]. Correlation of the depolarization field with the thickness of the interfacial layer, coercive field of FE and remanent polarization of the FE can be directly seen from (1). Furthermore,

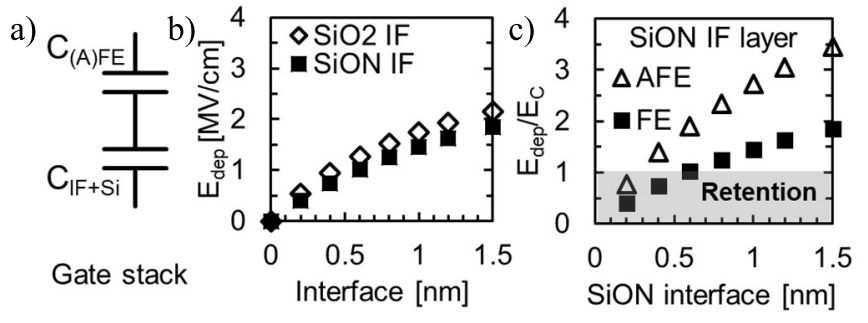

Fig. 8. Depolarization field as a function of the thickness of the IBL. (a) Equivalent model of the transistor gate stack comprising the capacitance of the $\mathrm{FE}$ or $\mathrm{AFE} \mathrm{C}_{(\mathrm{A}) \mathrm{FE}}$ and of the silicon space charge region and the interface layer combined, $\mathrm{C}_{\mathrm{IF}+\mathrm{Si}}$. (b) Depolarization field as a function of thickness of the interface layer for the different dielecric constants of the interface layer corresponding to $\mathrm{SiO}_{2}$ (open diamonds) and $\mathrm{SiON}$ (filled squares). (c) Depolarization field normalized to the coercive field as a function of the thickness of the interface layer for the case of a AFE (open triangles) and a FE (filled sqiures) switching layer.

it is important to note that due to the polycrystalline nature, non-uniformities or non-switching parts of the FE bulk, i.e., dead (non-switching) layers contributing to the depolarization, might have deteriorating impact on the polarization state and retention of the device. The depolarization field $\mathrm{E}_{\mathrm{dep}}$ is given by:

$$
E_{d e p}=\frac{P_{r}}{k_{F E} \varepsilon_{0}}\left(1+\frac{k_{I F} d_{F E}}{k_{F E} d_{I F}}\right)^{-1},
$$

where $\mathrm{P}_{\mathrm{r}}$ denotes the remanent polarization, $\mathrm{k}_{\mathrm{FE}}$ and $\mathrm{k}_{\mathrm{IF}}$ permittivity of the FE film and interface, respectively, while $\varepsilon_{0}$ represents the vacuum permittivity. In the following, three main ways to impact and decrease the depolarization field are described. All methods are based on engineering the voltage divider discussed in Section V-B. Voltage distribution can be changed by: 1) an increase of the k-value of the IBL (see Fig. 8b); 2) a decrease of the thickness of the IBL or 3) geometrical changes and employment of the internal gate electrode which could be used for tuning the area factor ratio between the FE and IBL [35].

As previously pointed out, the integration of the AFE material which is characterized by a lower $E_{c}$ and enables lower operation voltages, would tremendously reduce the stress over the IBL and extend the lifetime of the device. On the other side, lowering of the $\mathrm{E}_{\mathrm{c}}$ in case of usage of an AFE would have a detrimental impact on the MW as under the assumption of a large enough $\mathrm{P}_{\mathrm{r}}$ [36] the MW is expected to be directly proportional to $\mathrm{E}_{\mathrm{c}}$ of the used ferroelectric material. Moreover, not only does the MW reduce but the ratio between the $E_{\text {dep }} / E_{c}$ strongly rises as well. As a result, the retention of the device is heavily impacted (see Fig. 8c). From Fig. 8c it can be seen that an IBL thickness reduction bellow $0.3 \mathrm{~nm}$ would be mandatory to guarantee a stability of the stored state.

\section{Charge Trapping in Ferroelectric and Anti-Ferroelectric FETs}

As the last part of the comparison, stress (PRG/ERS) dependent charge trapping (CT) measurements are performed. The experiment is carried out as follows: a single pulse is applied to the gate of the transistor while its drain is biased at a voltage 
a)
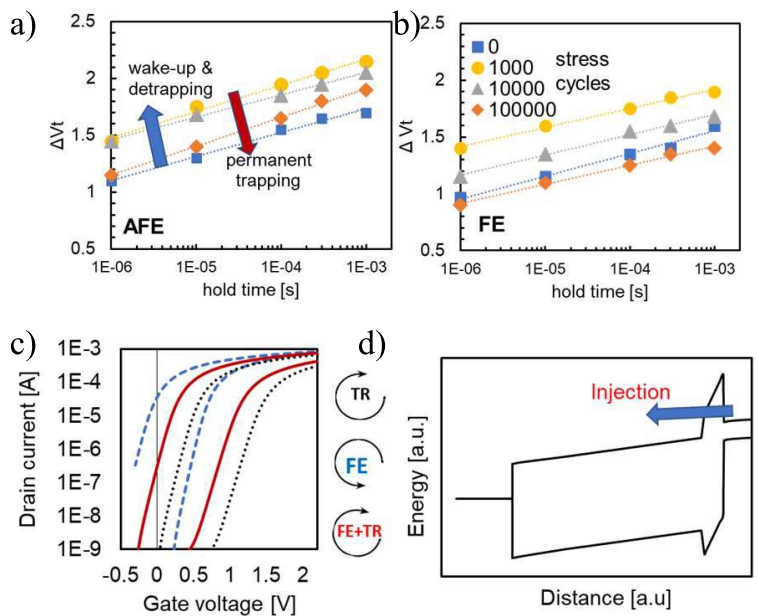

d)

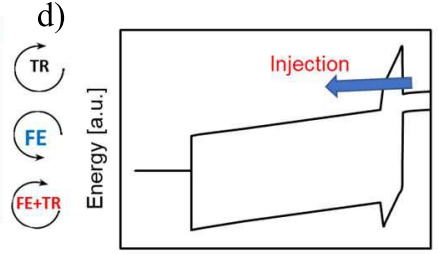

Distance [a.u]

e)

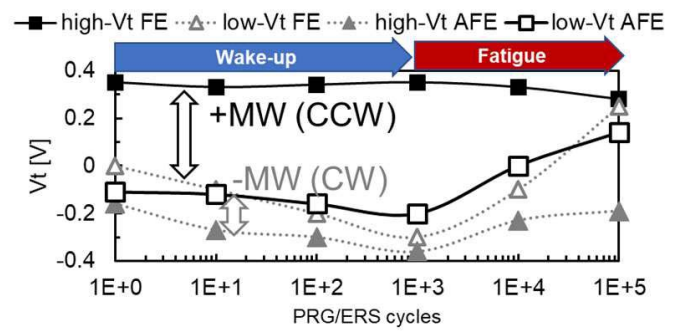

Fig. 9. a) PRG/ERS stress induced evolution of the $V_{t}$ shift as function of the pulse length of the charge trapping experiment measured on a) AFE FET and b) FeFET respectively. c) Simulated $I_{d}-V_{g}$ dual sweep previously residing in ERS state for a device for pure charge trapping(black), pure FE switching(blue) and combined chare trapping and FE swithcing (red); d) band diagram of FeFET with indicated charge injeciton. e) Endurance characteristic evolution of the PRG (low $V_{t}$ ) and ERS (high $V_{t}$ ) of a FeFET (squres and solid lines) and an AFE FET (triangles and dashed lines). The threshold voltages are extracted based on the constant current criterium. TR and FE denote trapping and ferroelectric swithing model respectively. The lines in e) are guides to the eyes.

of $100 \mathrm{mV}$ whereas source and bulk terminals are connected to ground. During the time when a single pulse is applied to the gate, gate voltage and drain current are recorded simultaneously by an oscilloscope. Subsequently, from the captured gate voltage $\left(\mathrm{V}_{\mathrm{g}}\right)$ and drain current $\left(\mathrm{I}_{\mathrm{d}}\right)$ transients the corresponding hysteretic $I_{d}-V_{g}$ characteristics are obtained. The difference between the threshold voltage recorded on the rising and falling edge is used to define a monitoring parameter, $\Delta \mathrm{V}_{\mathrm{t}}$. This single pulse based measurement is nested in cycling sequence required to monitor the evolution of the charge trapping behavior during the device lifetime. According to expectations, during the wake-up phase both FE and AFE based memory devices experienced an initially higher $\Delta \mathrm{V}_{\mathrm{t}}$ (see Figs. 9a-b). It should be noted that during the wake-up a low $\mathrm{V}_{\mathrm{t}}$ shifts towards more negative values which results in an opening of the MW. However, similar to the MW behavior with stress, after the wake-up stage the $\Delta \mathrm{V}_{\mathrm{t}}$ stays nearly constant and even a decrease of the $\Delta \mathrm{V}_{\mathrm{t}}$ is observed. This behavior can be explicitly seen in the case of the FeFET.

This surprising behavior can be explained using simulation to decouple the effects occurring within the FeFET stack. As a matter of fact, the $V_{t}$ of the FeFET depends on several components i.e., the amount of charge trapped within the stack, the amount of FE domains which increase during wake-up of the ferroelectric, defect generation rate due to the stressing and trapping and de-trapping kinetics. Therefore, a simulation of the CT experiment is performed in order to elucidate and explain the detrimental trapping kinetics within the FeFET. The TCAD Sentaurus device simulator from Synopsys and a FeFET model as introduced in Section V-B are used. Details about the model can be found in [21]. To decrease the complexity and increase the understanding of each component influencing the $\mathrm{V}_{\mathrm{t}}$, three scenarios are simulated: 1) a device with charge trapping only; 2) a device with FE switching and 3) a device with both trapping and FE switching combined. The antipodal nature of FE-switching and charge trapping can be understood upon application of a positive pulse on the gate of the transistor, where charge trapping results in a clockwise $(\mathrm{CW})$ hysteresis, whereas the FE switching results in a counter clock-wise (CCW) behavior (Fig. 9c). In the simulated device where both mechanisms are included, a $\mathrm{CCW}$ behavior is observable if the amount of the defects does not completely compensate the FE switching. As a second effect a trapped charge injected directly into the conduction band and later relaxed into the $\mathrm{HfO}_{2}$ (see Fig. 9d) shifts the low $\mathrm{V}_{\mathrm{t}}$ state towards more positive values as observed in the CT experiment (see Fig. 9c). As it can be seen from the endurance characteristics (Fig. 9e) of the FeFETs, the $\Delta \mathrm{V}_{\mathrm{t}}$ memory window is mainly affected by the change of the low $V_{t}$ value which is shifted by $0.3 \mathrm{~V}$ during cycling. This shift is added on the total $\Delta \mathrm{V}_{\mathrm{t}}$. Continuous PRG/ERS operations results in permanent trapping which compensates the FE switching resulting in a low $V_{t}$ shift and a closure of the MW (see Fig. 9e) and decrease of $\Delta \mathrm{V}_{\mathrm{t}}$. Compared to the $\mathrm{FE}$ transistor which is characterized by a $\mathrm{CCW}$ behavior even in pristine stage, the AFE device performs in a CW behavior with a "negative' FE MW. As the remanent polarization of the AFE device is low completely trapped device behavior accompanied with $\mathrm{CW}$ hysteresis is observable. Independently on the 'negative' MW, during the initial $10^{3}$ cycles a behavior similar to a wakeup (decrease of low- $\mathrm{V}_{\mathrm{t}}$; see Fig. 9e) is observable due to the de-trapping which consequently contributes to an increase of $\Delta \mathrm{V}_{\mathrm{t}}$ observed in $\mathrm{CT}$ tests. Equivalent to a FeFET a continuous stressing of the AFeFET results in a permanent trapping which consequently decreases the $\Delta \mathrm{V}_{\mathrm{t}}$.

\section{CONCLUSiON}

Stabilization of ferroelectric and anti-ferroelectric properties in simple binary oxides offers scalability and CMOS compatibility of ferroelectric memories with state-of-the-art technology nodes. However, in contrast to PZT memories, binary oxide based FE solutions suffer from limited endurance typically disrupted by hard breakdown caused by the high Ec value. On the other side, implementation of a $\mathrm{ZrO}_{2}$ based AFE material in a 1T-1C type memory configuration results in a nonvolatile memory with tremendous increase in endurance compared to the FE binary oxide counterpart. In this paper, a comparative study of FE and AFE based memories focusing on both $1 \mathrm{~T}$ and 1T-1C solutions is performed. We show DRAM like endurance of AFE based capacitors that 
is orders of magnitude higher with respect to the FE equivalent. Secondly, a reduced switching field required for state change of AFE-RAM further decreases the operation voltage and hence, stress-induced degradation, enabling a low power non-volatile memory. On the other side, a reduction of the switching barrier in AFE memories results in a lower retention and temperature immunity of an AFE-RAM compared to FeRAM.

Nonetheless, the use of a polarized and non-polarized state in AFE-RAM confines shift of the coercive field only for one state in contrast to a parallel shift of the complete hysteresis loop in FE memories. Finally, a physical thickness increase of the anti-ferroelectric of only $2 \mathrm{~nm}$ compared to dielectric thickness used in state-of-the-art DRAM nodes would satisfy the 10 -year reliability specification and allow operation voltages in the order of $1.2 \mathrm{~V}$.

In the second part of the paper, a detailed reliability analysis of a FE vs. AFE based single transistor is performed. We show that the usage of AFE and accompanying reduction of switching field would effectively reduce the field stress over the interface buffer layer, compared to the FE counterpart. Nonetheless, the reduction of the coercive field would not only decrease the width of the memory window but also increase the depolarization field which is detrimental for the retention performance of the device.

\section{ACKNOWLEDGMENT}

This work was financially supported by the European Fund for regional Development EFRD and the Free State of Saxony (Germany), Europe supports Saxony. We acknowledge support and FeFET hardware provided by GLOBALFOUNDRIES Fab1 LLC.

\section{REFERENCES}

[1] K. Kim and Y. J. Song, "Integration technology for ferroelectric memory devices," Microelectron. Rel., vol. 43, no. 3, pp. 385-398, Mar. 2003, doi: 10.1016/S0026-2714(02)00285-8.

[2] S. R. Summerfelt et al., "High-density 8Mb 1T-1C ferroelectric random access memory embedded within a low-power $130 \mathrm{~nm}$ logic process," in Proc. 6th IEEE Int. Symp. Appl. Ferroelect., Nara, Japan, 2007, pp. 9-10, doi: 10.1109/ISAF.2007.4393151.

[3] T. S. Böscke, J. Müller, D. Bräuhaus, U. Schröder, and U. Böttger, "Ferroelectricity in hafnium oxide thin films," Appl. Phys. Lett., vol. 99, no. 10 , pp. 1-3, Sep. 2011, doi: 10.1063/1.3634052.

[4] M. Hoffmann et al., "Direct observation of negative capacitance in polycrystalline ferroelectric $\mathrm{HfO}_{2}$," Adv. Funct. Mater, vol. 26, no. 47, pp. 8643-8649, Oct. 2016, doi: 10.1002/adfm.201602869.

[5] A. I. Khan et al., "Negative capacitance in a ferroelectric capacitor," Nat. Mater, vol. 14, no. 2, pp. 182-186, Feb. 2015, doi: 10.1038/nmat4148.

[6] S. Salahuddin and S. Datta, "Use of negative capacitance to provide voltage amplification for low power nanoscale devices," Nano Lett., vol. 8, no. 2, pp. 405-410, Dec. 2008, doi: 10.1021/n1071804g.

[7] H. Mulaosmanovic et al., "Novel ferroelectric FET based synapse for neuromorphic systems," in Proc. Symp. VLSI Technol., Kyoto, Japan, 2017, pp. T176-T177, doi: VLSIT.2017.7998165.

[8] M. T. Bohr, R. S. Chau, T. Ghani, and K. Mistry, "The highk solution," IEEE Spectr, vol. 44, no. 10, pp. 29-35, Oct. 2007, doi: 10.1109/MSPEC.2007.4337663.

[9] J. Muller et al., "Ferroelectric hafnium oxide: A CMOS-compatible and highly scalable approach to future ferroelectric memories," in Proc. IEEE Int. Electron Devices Meeting, Washington, DC, USA, 2013, pp. 10.8.1-10.8.4, doi: 10.1109/IEDM.2013.6724605.
[10] M. Trentzsch et al., "A 28nm HKMG super low power embedded NVM technology based on ferroelectric FETs," in Proc. IEEE Int. Electron Devices Meeting (IEDM), San Francisco, CA, USA, 2016, pp. 11.5.1-11.5.4, doi: 10.1109/IEDM.2016.7838397.

[11] E. Yurchuk et al., "Charge-trapping phenomena in $\mathrm{HfO}_{2}$-based FeFETtype nonvolatile memories," IEEE Trans. Electron Devices, vol. 63, no. 9, pp. 3501-3507, Sep. 2016, doi: 10.1109/TED.2016.2588439.

[12] M. Pesic et al., "Root cause of degradation in novel $\mathrm{HfO}_{2}$ based ferroelectric memories," in Proc. IEEE Int. Rel. Phys. Symp. (IRPS), Pasadena, CA, USA, 2016, pp. MY-3-1-MY-3-5, doi: 10.1109/IRPS.2016.7574619.

[13] M. Pešić et al., "Physical mechanisms behind the field-cycling behavior of $\mathrm{HfO}_{2}$-based ferroelectric capacitors," Adv. Funct. Mater, vol. 26 no. 25, pp. 4601-4612, Jul. 2016, doi: 10.1002/adfm.201600590.

[14] E. Yurchuk et al., "Origin of the endurance degradation in the novel $\mathrm{HfO}_{2}$-based $1 \mathrm{~T}$ ferroelectric non-volatile memories," in Proc. IEEE Int. Rel. Phys. Symp., Waikoloa, HI, USA, 2014, pp. 2E.5.1-2E.5.5, doi: 10.1109/IRPS.2014.6860603.

[15] M. Pešić, M. Hoffmann, C. Richter, T. Mikolajick, and U. Schroeder, "Nonvolatile random access memory and energy storage based on antiferroelectric like hysteresis in $\mathrm{ZrO}_{2}$," Adv. Funct. Mater., vol. 26, no. 41, pp. 7486-7494, Nov. 2016, doi: 10.1002/adfm.201603182.

[16] M. Pesic et al., "How to make DRAM non-volatile? Anti-ferroelectrics: A new paradigm for universal memories," in Proc. IEEE Int. Electron Devices Meeting (IEDM), San Francisco, CA, USA, 2016, pp. 116.1-11.6.4, doi: 10.1109/IEDM.2016.7838398.

[17] M. Pesic et al., "Anti-ferroelectric $\mathrm{ZrO}_{2}$, an enabler for low power non-volatile 1T-1C and 1T random access memories," in Proc. 47th Eur. Solid-State Device Res. Conf. (ESSDERC), Leuven, Belgium, 2017, pp. 160-163, doi: 10.1109/ESSDERC.2017.8066616.

[18] T. Schloesser et al., "6F2 buried wordline DRAM cell for 40nm and beyond," in Proc. IEEE Int. Electron Devices Meeting, San Francisco, CA, USA, 2008, pp. 1-4, doi: 10.1109/IEDM.2008.4796820.

[19] J. Müller et al., "Ferroelectricity in simple binary $\mathrm{ZrO}_{2}$ and $\mathrm{HfO}_{2}$," Nano Lett., vol. 12, no. 8, pp. 4318-4323, Jul. 2012, doi: 10.1021/nl302049.

[20] M. Pesic, Gate Stack Engineering for Emerging Polarization Based NonVolatile Memories. Norderstedt, Germany: Books Demand, 2017.

[21] R. Waser, Nanoelectronics and Information Technology. Weinheim, Germany: Wiley, 2012.

[22] E. D. Grimley et al., "Structural changes underlying field-cycling phenomena in ferroelectric $\mathrm{HfO}_{2}$ thin films," Adv. Electron. Mater, vol. 2, no. 9, pp. 1600173-1600177, Sep. 2016, doi: 10.1002/aelm.201600173.

[23] T. Schenk et al., "Complex internal bias fields in ferroelectric hafnium oxide," ACS Appl. Mater. Inter., vol. 7, no. 36, pp. 20224-20233, Aug. 2015, doi: 10.1021/acsami.5b05773.

[24] U. Schroeder et al., "Impact of field cycling on $\mathrm{HfO}_{2}$ based nonvolatile memory devices," in Proc. 46th Eur. Solid-State Device Res. Conf. (ESSDERC), Lausanne, Switzerland, 2016, pp. 364-368, doi: 10.1109/ESSDERC.2016.7599662.

[25] C. Künneth et al., "Modeling ferroelectric film properties and size effects from tetragonal interlayer in $\mathrm{Hf}_{1}-x \mathrm{Zr}_{x} \mathrm{O}_{2}$ grains," J. Appl. Phys., vol. 121, no. 20, pp. 205304-205308, May 2017, doi: $10.1063 / 1.4983811$.

[26] J. Rodriguez et al., "Reliability of ferroelectric random access memory embedded within $130 \mathrm{~nm}$ CMOS," in Proc. IEEE Int. Rel. Phys. Symp., Anaheim, CA, USA, Dec. 2010, pp. 750-758, doi: 10.1109/IRPS.2010.5488738.

[27] S. Mueller, J. Muller, U. Schroeder, and T. Mikolajick, "Reliability characteristics of ferroelectric $\mathrm{Si}: \mathrm{HfO}_{2}$ thin films for memory applications," in Proc. IEEE Trans. Device Mater. Rel., vol. 13, no. 1, pp. 93-97, Mar. 2013, doi: 10.1109/TDMR.2012.2216269.

[28] M. Pešić et al., "Anti-ferroelectric-like $\mathrm{ZrO}_{2}$ non-volatile memory: Inducing non-volatility within state-of-the-art DRAM," in Proc. 17th IEEE Non-Volatile Memory Technol. Symp. (NVMTS), Aachen, Germany, 2017, pp. 1-4, doi: 10.1109/NVMTS.2017.8171307.

[29] M. Pešić et al., "Low leakage $\mathrm{ZrO}_{2}$ based capacitors for sub $20 \mathrm{~nm}$ dynamic random access memory technology nodes," J. Appl. Phys., vol. 119, no. 6, pp. 064101-064106, Jan. 2016, doi: 10.1063/1.4941537.

[30] S. Knebel et al., "Reliability comparison of $\mathrm{ZrO}_{2}$-based DRAM high-k dielectrics under DC and AC stress," IEEE Trans. Device Mater. Rel., vol. 17, no. 2, pp. 324-330, Jun. 2017, doi: 10.1109/TDMR.2017.2699287.

[31] S. Riedel, P. Polakowski, and J. Müller, "A thermally robust and thickness independent ferroelectric phase in laminated hafnium zirconium oxide," AIP Adv., vol. 6, no. 9, pp. 095123-095127, Sep. 2016, doi: $10.1063 / 1.4964300$. 
[32] M. Pešić et al., "A computational study of hafnia-based ferroelectric memories: From ab initio via physical modeling to circuit models of ferroelectric device," J. Comput. Elect., vol. 16, no. 4, pp. 1236-1256, Dec. 2017, doi: 10.1007/s10825-017-1053-0.

[33] M. Pešić, U. Schroeder, S. Slesazeck, and T. Mikolajick, "Reliability aspects of novel anti-ferroelectric non-volatile memories compared to hafnia based ferroelectric memories," in Proc. IEEE Int. Integr. Rel. Workshop (IIRW), South Lake Tahoe, CA, USA, 2017.

[34] T. P. Ma and J.-P. Han, "Why is nonvolatile ferroelectric memory fieldeffect transistor still elusive?" IEEE Electron Device Lett., vol. 23, no. 7, pp. 386-388, Jul. 2002, doi: 10.1109/LED.2002.1015207.

[35] J. Muller et al., "High endurance strategies for hafnium oxide based ferroelectric field effect transistor," in Proc. 16th IEEE Non-Volatile Memory Technol. Symp. (NVMTS), Pittsburgh, PA, USA, Dec. 2016, pp. 1-7, doi: 10.1109/NVMTS.2016.7781517.

[36] S. L. Miller and P. J. McWhorter, "Physics of the ferroelectric nonvolatile memory field effect transistor," J. Appl. Phys., vol. 72, no. 12, pp. 5999-6010, Sep. 1992, doi: 10.1063/1.351910.

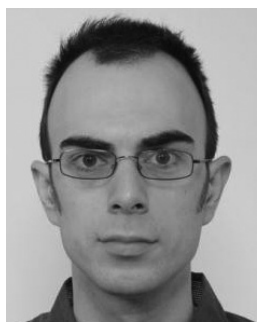

Milan Pešić received the Ph.D. degree in 2017 from the Technical University of Dresden, Germany. His research activities are in the field of emerging non-volatile memories and devices. Particularly, his interests are the high-k devices, ferroelectrics, electrical characterization and modeling of emerging NVMs as well as the energy efficient electronics (steep-slope device). In February 2018 he joined MDLSoft Inc., a Silicon Valley company developing software solutions for the simulation of micro/nano-electronic devices and Ferroelectric Memory Company developing ferroelectric memories. Previously he was with NaMLab, Dresden, Germany. Up to now, he authored and coauthored more than 30 technical papers and patent.

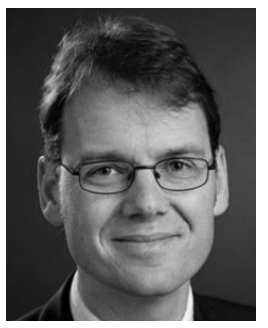

Uwe Schroeder received his doctoral degree at University of Bonn, Germany including a research visit at UC Berkeley and worked at University of Chicago as a post-doctoral researcher. $\mathrm{He}$ joined Infineon formerly Siemens Semiconductor in 1997 for capacitor development in the DRAM Development Alliance with IBM, before transferring to Infineon's Memory Development Center in Dresden, Germany in 2000. There, he continued the research on high-k dielectrics and their integration into DRAM capacitors. During this time the ferroelectric properties of $\mathrm{HfO} 2$ based dielectrics were found. In 2009 he moved to NaMLab and pursued his work on high-k dielectrics and ferroelectric $\mathrm{HfO}_{2}$-layers.

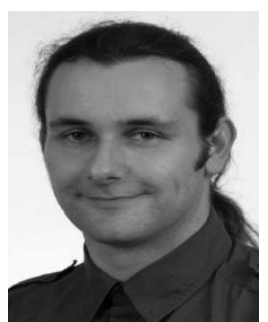

Stefan Slesazeck is a Senior Scientist at NaMLab responsible for concept evaluation, hardware development, electrical characterization and modelling of memories. One of his research interests is the development of novel computing paradigms based on memristors. Prior to NaMLab, he was a project leader for the pre-development of new memory concepts with Qimonda Dresden (Germany) focusing on concept evaluation for 1T - DRAM including floating body devices, cell concepts, access schemes for WL-driver and sense amplifier. As a device engineer at Infineon Technologies, Stefan focused on the module development of 3D DRAM access devices in $65 \mathrm{~nm}$ and $46 \mathrm{~nm}$ buried word line technology and pre-development of 3D DRAM access devices as FinFET and EUD. Stefan received his Ph.D. at TU Dresden.

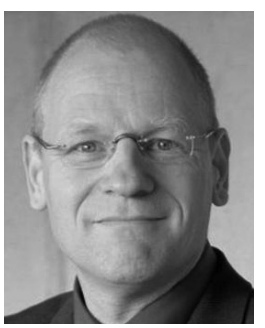

Thomas Mikolajick received the Diploma S(Dipl.-Ing.) in electrical engineering in 1990 and his $\mathrm{PhD}$ in electrical engineering in 1996 both from the University Erlangen-Nuremberg. From 1996 until 2006 he was in the semiconductor industry developing CMOS processes, ferroelectric memories, emerging non-volatile memories and Flash memories first at Siemens Semiconductor and later at Infineon. In late 2006 he moved back to academia taking over a professorship for material science of electron devices and sensors at the University of Technology Freiberg. In October 2009 he started at Technische Universität Dresden were he now holds a professorship for nanoelectronic materials in combination with the position of scientific director at NaMLab GmbH. Prof. Mikolajick is author or co-author of more than 350 Publications in scientific journals or at scientific conferences and inventor or co-inventor of about 50 patents. 